\title{
Boussignac CPAP system for brain death confirmation with apneic test in case of acute lung injury/adult respiratory distress syndrome - series of cases
}

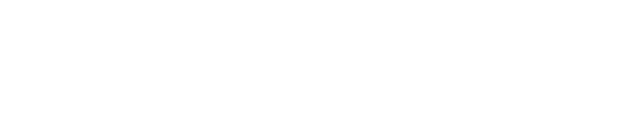

\author{
Andrzej Wieczorek' \\ Tomasz Gaszynski² \\ 'Department of Anesthesia and \\ Intensive Care, Medical University \\ of Lodz, Lodz, Poland; '2Department \\ of Emergency Medicine and Disaster \\ Medicine, Medical University of Lodz, \\ Lodz, Poland
}

Introduction: There are some patients with severe respiratory disturbances like adult respiratory distress syndrome (ARDS) and suspicion of brain death, for whom typical performance of the apneic test is difficult to complete because of quick desaturation and rapid deterioration without effective ventilation. To avoid failure of brain death confirmation and possible loss of organ donation another approach to apneic test is needed. We present two cases of patients with clinical symptoms of brain death, with lung pathology (acute lung injury, ARDS, lung embolism and lung infection), in whom apneic tests for recognizing brain death were difficult to perform. During typical performance of apneic test involving the use of oxygen catheter for apneic oxygenation we observed severe desaturation with growing hypotension and hemodynamic destabilization. But with the use of Boussignac CPAP system all necessary tests were successfully completed, confirming the patient's brain death, which gave us the opportunity to perform procedures for organ donation. The main reason of apneic test difficulties was severe gas exchange disturbances secondary to ARDS. Thus lack of positive end expiratory pressure during classical performance of apneic test leads to quick desaturation and rapid hemodynamic deterioration, limiting the observation period below dedicated at least 10-minute interval.

Conclusion: The Boussignac CPAP system may be an effective tool for performing transparent apneic test in case of serious respiratory disturbances, especially in the form of acute lung injury or ARDS.

Keywords: brain death, organ donor, ARDS, ALI, Boussignac CPAP

\section{Introduction}

In many countries there is a growing problem of organ shortage for transplantation. Many initiatives have been introduced all over the world to improve both the qualification process and the accessibility of potential organ donors. ${ }^{1-3}$ Although there is large variability between different countries and centers regarding brain death determination criteria ${ }^{4,5}$ the apneic test for confirmation of the irreversible lack of spontaneous respiratory pattern is one of the most commonly accepted and performed tests. Typical performance of this test involves the use of an oxygen catheter for apneic oxygenation of the patient's lungs to avoid possible hypoxia during the procedure. This technique of apneic oxygenation has some limitations ${ }^{6,7}$ but in most cases is an effective tool for sufficient oxygenation of the patient. However, there are some patients with severe respiratory disturbances and suspicion of brain death, for whom typical performance of the apneic test is difficult to complete because of quick desaturation and rapid deterioration without effective ventilation. To avoid failure of brain death confirmation and
Correspondence: Tomasz Gaszynsk Department of Emergency Medicine and Disaster Medicine, Medical University of Lodz, Kopcinskiego 22, Lodz 90 - I53, Poland

Tel +4842 6783748

Email tomasz.gaszynski@umed.lodz.pl 
possible organ donation loss there is a necessity for the use of another approach to the apneic test. ${ }^{8}$ The alternative protocols for apneic test were previously successfully checked, with the use of CPAP system for patients with oxygenation difficulties. ${ }^{9}$ Additionally the application of CPAP with the use of anesthesia machine circuit system ${ }^{10}$ or CPAP valve with recruitment maneuver ${ }^{11}$ were prescribed for the stabilization of oxygenation during apneic test. We implemented an alternative method with the use of Boussignac virtual valve (Boussignac CPAP system, Vygon, Ecouen, France) for positive end expiratory pressure (PEEP) creation during apneic test. The Boussignac CPAP system was previously successfully applied in clinical use in cases of spontaneously breathing patients with respiratory difficulties. ${ }^{12-14}$ The main advantages of this method are simple construction of the small device and lack of possibility for artificially created ventilation, which is important for transparency of apneic test and effectiveness in functional residual capacity (FRC) stabilization.

\section{Case I report}

The 36-year old patient was admitted to the intensive care unit (ICU) because of respiratory insufficiency during severe intracranial hemorrhage. He initially presented signs of acute lung injury, probably because of previous gastric content aspiration during initial period of neurological deterioration, evolving into full scale adult respiratory distress syndrome (ARDS) (Figure 1). After patient stabilization with the use of ventilation therapy and catecholamine infusions we observed progressive deterioration of central nervous system functions, including deep unconsciousness and loss of all brainstem functions, indicating possible brain death.

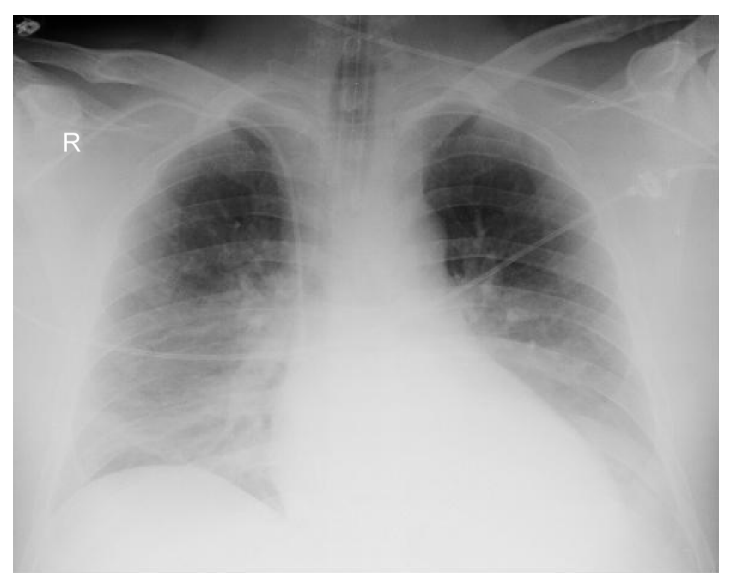

Figure I X-ray of chest of first case - ARDS following massive gastric content aspiration.

Abbreviation: ARDS, adult respiratory distress syndrome.
To confirm the suspicion of brain death we completed the law regulated diagnostic protocol for brain death diagnosis, including two apneic tests performed with appropriate time interval. It involved prerequisite fulfillment: after the period of observation confirmation of deep comatose state with diagnosed origin, presence of full scale artificial ventilation without spontaneous efforts, confirmation of irreversible brain damage and exclusion of toxicological disorders, hypothermia $35^{\circ} \mathrm{C}$ and below, and severe metabolic and endocrine disorders. The next part of the procedure involves two sets of tests performed with appropriate to the clinical situation time interval. The tests consist of positive confirmation of lack of brain stem reflexes and functions: corneal reflex, both spontaneous eye movement and eye movement during caloric test, brain-eye reflex, iris reaction to light, coughing and vomiting reflexes, respiratory pattern, and lack of reaction to pain stimuli. According to the Polish guidelines, before the apneic test initiation the patient was pre-oxygenated during mechanical ventilation with the use of $\mathrm{FiO}_{2}=1,0$ through 10 minutes. The mechanical parameters of mechanical ventilation were established to achieve $\mathrm{PaCO}_{2}$ value of at least $40 \mathrm{mmHg}$, avoiding hyperventilation. During typical performance of this test involving the use of oxygen catheter for apneic oxygenation we observed severe desaturation with growing hypotension and hemodynamic destabilization, creating risk of immediate cardiac arrest (first test). There were no observed respiratory efforts, or any other spontaneous activity of the patient, but quick desaturation and rapid deterioration with both speeds of oxygen delivery $6 \mathrm{~L} / \mathrm{min}$ and $15 \mathrm{~L} / \mathrm{min}$ which had created the need for return to mechanical ventilation, without enough long observation period for proper assessment. To eliminate all possible concerns about achieved results we performed additional apneic tests with the use of Boussignac CPAP system (Figure 2). The use of Boussignac CPAP system with oxygen flow $15 \mathrm{~L} / \mathrm{min}$ created enough stabilization for apneic test performance without circulatory deterioration. During apneic tests performed with the use of Boussignac virtual valve we observed much smaller disturbances, with stable hemodynamic function and much more acceptable saturation (second test). Between the series of tests panangiography was performed, which confirmed total lack of cerebral blood flow. All necessary tests were successfully completed, confirming the patient's death, which gave us the opportunity to perform procedure for organ donation. ${ }^{15}$ The main reason for apneic test difficulties was severe gas exchange disturbances secondary to ARDS. During previous period of ventilation the patient needed advanced 


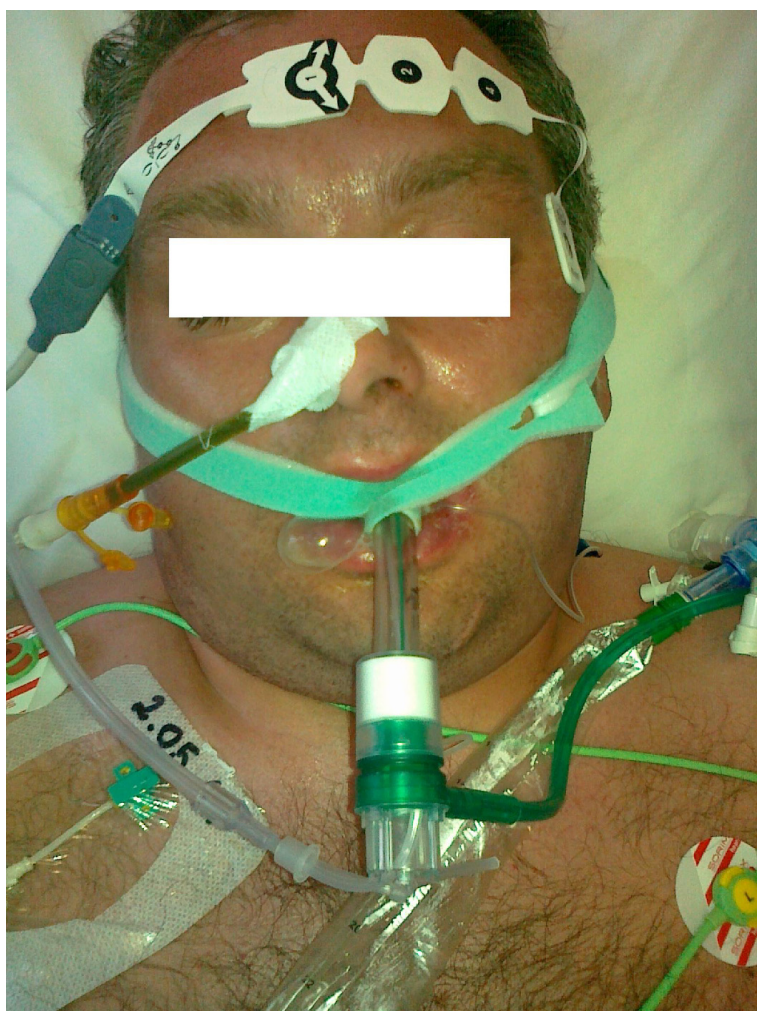

Figure 2 Presentation of Boussignac CPAP (Vygon, Ecouen, France) system during apneic test.

ventilation protocol with high PEEP value (at least $15 \mathrm{~cm}$ $\left.\mathrm{H}_{2} \mathrm{O}\right)$ to avoid quick atelectasis formation. Thus, lack of PEEP during classical performance of apneic test led to quick desaturation and rapid hemodynamic deterioration, limiting the observation period to less than the minimum 10-minute interval. Apneic tests with the Boussignac CPAP system showed lack of any observed or monitored spontaneous respiratory effort or activity. Laboratory gas analysis results were: first test (typical apneic test with oxygen insufflation only): $\mathrm{pH} 7.361 / 7.157 ; \mathrm{PaCO}_{2} 52.9 / 93.8$ mmHg; $\mathrm{PaO}_{2} 72.3 / 52.5$ mmHg; $\mathrm{SaO}_{2}$ 94.7/78.6\%; second test (with Boussignac system): $\mathrm{pH}$ 7.338/7.11; $\mathrm{PaCO}_{2}$ 56.8/108 mmHg; $\mathrm{PaO}_{2} 118 / 78.1 \mathrm{mmHg} ; \mathrm{SaO}_{2}$ 98.2/91.1\%. These data completed the diagnostic protocol confirming the patient's brain death status.

\section{Case 2 report}

A 39-year old woman was admitted to internal ward because of severe respiratory distress. She complained of breathing difficulty for 2 days, which deteriorated and while presenting to the emergency department she was getting tired quickly and required oxygen supply to maintain $\mathrm{SpO}_{2}>90 \%$. Chest $\mathrm{X}$-rays revealed lung embolism complicated by lung infection (Figure 3). After thrombosis therapy and antibiotics her state

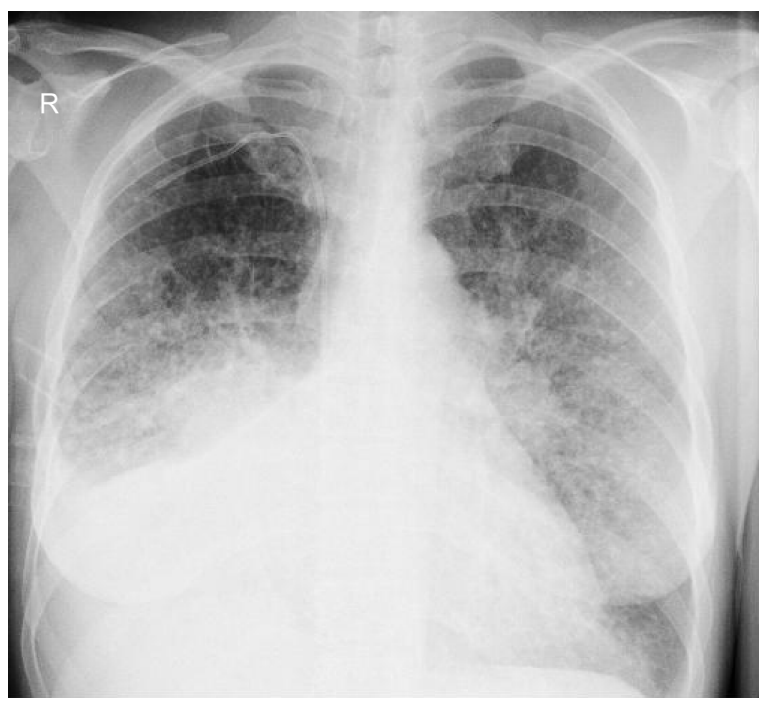

Figure $3 \mathrm{X}$-ray of chest of second patient - ARDS after lung embolism complicated by lung infection.

Abbreviation: ARDS, adult respiratory distress syndrome.

improved slightly. On the third day of hospital stay she had sudden respiratory failure and cardiac arrest. After successful resuscitation computed tomography scans of head revealed intracranial bleeding. After admission to ICU she presented signs of brain death. After 24 hours' observation, tests for determining brain death were started. The patient's lungs were ventilated with $100 \%$ oxygen to achieve $40 \mathrm{mmHg}$ in $\mathrm{EtCO}_{2}$ (Figure 4). First apneic test was performed with typical method - passive oxygen insufflation via catheter in endotracheal tube. This test was very difficult to perform because of fast desaturation. We decided to perform second apneic test with the use of Boussignac CPAP system (Figure 5). This test was effective and did not result in desaturation nor hemodynamic instability.

The laboratory gas analysis results were: first test (typical apneic test with oxygen insufflation only): $\mathrm{pH} 7.257 /$ 6.99; $\mathrm{PaCO}_{2}$ 58.2/117 mmHg; $\mathrm{PaO}_{2} 90.7 / 83.5 \mathrm{mmHg}$; $\mathrm{SaO}_{2}$ 92.9/87.9\%; second test (with Boussignac system): pH 7.11/6.98; $\mathrm{PaCO}_{2}$ 74/101 mmHg; $\mathrm{PaO}_{2}$ 97/92 mmHg; $\mathrm{SaO}_{2}$ 99.6/96.2\%.

\section{Discussion}

The number of reports involving use of different techniques of PEEP generation during apneic test for FRC and oxygenation stabilization is very limited. Thus there is no standard procedure available for such a clinical situation. On the other hand the apneic test is one of the crucial elements necessary for brain death confirmation and one of the most commonly accepted and performed. Poland as well as a few 


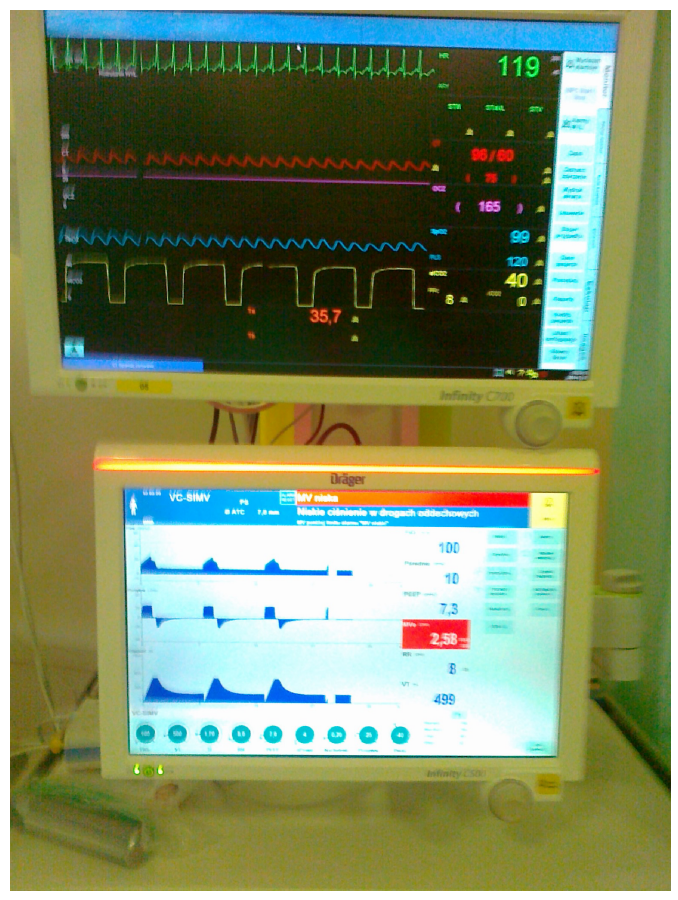

Figure 4 Picture of monitors - at the start of apneic test achieved $\mathrm{EtCO}_{2}$ was $40 \mathrm{mmHg}$, but in blood gasometry $\mathrm{PaCO}_{2}$ was $74 \mathrm{mmHg}$.

Note: Drager Infinity C 700 (upper monitor, showing among other parameters $\mathrm{EtCO}_{2}$ - yellow curve and numeric value) and Drager Infinity C 500 for Evita E ventilator (lower panel showing ventilation curves before start point for patient disconnection from ventilator and apneic test).

other countries introduced this test into the law regulated diagnostic protocol for brain death confirmation. In case of unstable patients with severe respiratory disturbances very rarely is there a possibility for successful procedure with the use of oxygen catheter alone, because of deep desaturation and hemodynamic destabilization occurrence. Many different techniques were proposed for such a situation to stabilize FRC and gas exchange. ${ }^{9-11}$ The advantages of Boussignac

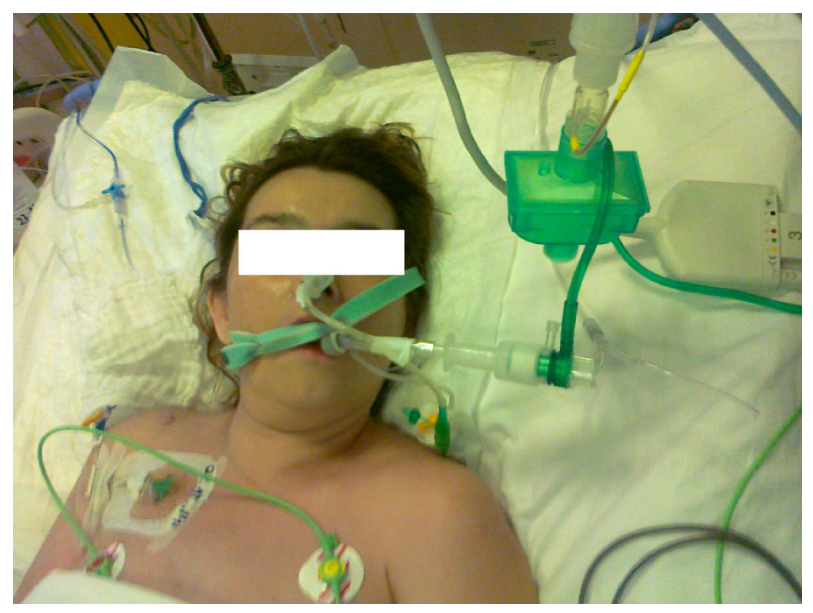

Figure 5 Patient during second series of apneic test using Boussignac CPAP system (Vygon, Ecouen, France).
CPAP device in such a situation are low costs, simple construction of the device, possibility for use together with classical oxygen catheter, and lack of possibility for artificially created ventilation, which is important for transparency of apneic test. Although the last measurements of oxygenation from the second test were below optimal objective, the tests were successfully completed without hemodynamic decompensation. However, this single observation is only a small indication of possible benefits from improved technique of apneic test. Further studies, involving a bigger population of patients, should be performed to establish real value of this improvement of apneic test technique.

\section{Conclusion}

The Boussignac CPAP system may be an effective tool for transparent performance of apneic tests in case of serious respiratory disturbances, especially in the form of acute lung injury or ARDS. A randomized multicenter study may be necessary to compare different proposed techniques for such a clinical situation.

\section{Disclosure}

The authors have no conflicts of interest to disclose.

\section{References}

1. Gupta N, Garonzik-Wang JM, Passarella RJ, et al. Assessment of resident and fellow knowledge of the organ donor referral process. Clin Transplant. 2014;28(4):443-449.

2. Neyrinck A, Van Raemdonck D, Monbaliu D. Donation after circulatory death: current status. Curr Opin Anaesthesiol. 2013;26(3):382-390.

3. Shemie SD, Hornby L, Baker A, et al. International guideline development for the determination of death. Intensive Care Med. 2014;40(6): 788-797.

4. Wijdicks EF. Brain death. Handb Clin Neurol. 2013;118:191-203.

5. Shappell CN, Frank JI, Husari K, Sanchez M, Goldenberg F, Ardelt A. Practice variability in brain death determination: a call to action. Neurology. 2013;81(23):2009-2014.

6. Olguner C, Koca U, Akan M, Karci A, Elar Z. The safe limits of mechanical factors in the apnea testing for the diagnosis of brain death. Tohoku J Exp Med. 2007;211(2):115-120.

7. Henry NR, Marshall SG. Apnea testing: the effects of insufflation catheter size and flow on pressure and volume in a test lung. Respir Care. 2014;59(3):406-410.

8. Del Río F, Escudero D, De La Calle B, Vidal FG, Paredes MV, Núñez JR. Evaluación y mantenimiento del donante pulmonar [Evaluation and maintenance of the lung donor]. Med Intensiva. 2009; 33(1):40-49. Spanish.

9. Lévesque S, Lessard MR, Nicole PC, et al. Efficacy of a T-piece system and a continuous positive airway pressure system for apnea testing in the diagnosis of brain death. Crit Care Med. 2006;34(8):2213-2216.

10. Shrestha GS, Shrestha PS, Acharya SP, et al. Apnea testing with continuous positive airway pressure for the diagnosis of brain death in a patient with poor baseline oxygenation status. Indian J Crit Care Med. 2014;18(5):331-333.

11. Hocker S, Whalen F, Wijdicks EF. Apnea testing for brain death in severe acute respiratory distress syndrome: a possible solution. Neurocrit Care. 2014;20(2):298-300. 
12. Dieperink W, Jaarsma T, van der Horst IC, et al. Boussignac continuous positive airway pressure for the management of acute cardiogenic pulmonary edema: prospective study with a retrospective control group. BMC Cardiovasc Disord. 2007;7:40.

13. Wong DT, Adly E, Ip HY, Thapar S, Maxted GR, Chung FF. A comparison between the Boussignac ${ }^{\mathrm{TM}}$ continuous positive airway pressure mask and the venturi mask in terms of improvement in the $\mathrm{PaO} 2 /$ $\mathrm{F}(\mathrm{I}) \mathrm{O} 2$ ratio in morbidly obese patients undergoing bariatric surgery: a randomized controlled trial. Can J Anaesth. 2011;58(6):532-539.
14. Moritz F, Brousse B, Gellée B, et al. Continuous positive airway pressure versus bilevel noninvasive ventilation in acute cardiogenic pulmonary edema: a randomized multicenter trial. Ann Emerg Med. 2007;50(6): 666-675.

15. Trzcinska M, Woderska A, Wlodarczyk Z. Psychological principles in regard to interview with the deceased donor's family. Anaesthesiol Intensive Ther. 2014;47:200-207.

\section{Publish your work in this journal}

Therapeutics and Clinical Risk Management is an international, peerreviewed journal of clinical therapeutics and risk management, focusing on concise rapid reporting of clinical studies in all therapeutic areas outcomes, safety, and programs for the effective, safe, and sustained use of medicines. This journal is indexed on PubMed Central, CAS,
EMBase, Scopus and the Elsevier Bibliographic databases. The manuscript management system is completely online and includes a very quick and fair peer-review system, which is all easy to use. Visit http://www.dovepress.com/testimonials.php to read real quotes from published authors.

Submit your manuscript here: http://www.dovepress.com/therapeutics-and-clinical-risk-management-journal 